\title{
Probing the Desert with Fermion Masses
}

\author{
Jonathan Bagger \\ Stanford Linear Accelerator Center, Stanford University, Stanford, California 94305
}

Savas Dimopoulos

Department of Physics, Stanford University, Stanford, California 94305

and

Eduard Massó ${ }^{(a)}$

Stanford Linear Accelerator Center, Stanford University, Stanford, California 94305

(Received 2 August 1985)

\begin{abstract}
We use the $S U(3) \otimes S U(2) \otimes U(1)$ renormalization-group equations to place upper limits on the scale where new physics beyond the standard model must emerge. Our bounds rely solely on the structure of the renormalization-group equations and on the magnitudes of heavy-quark masses. For example, if $\Sigma m_{q}^{2} \geqslant(450 \mathrm{GeV})^{2}$, new physics must be found below $10^{5} \mathrm{GeV}$.
\end{abstract}

PACS numbers: $12.10 . \mathrm{En}, 11.10 . \mathrm{Gh}$

The standard $S U(3) \otimes S U(2) \otimes U(1)$ model provides a very successful description of the strong, weak, and electromagnetic interactions. It is, however, in no sense a fundamental theory. The SU(3) $\otimes S U(2)$ $\otimes U(1)$ model contains at least eighteen parameters, each of which must be adjusted by hand to account for low-energy phenomenology.

What lies beyond the standard model? A variety of extensions have been discussed in recent years. Most of these proposals predict new physics above a characteristic scale $M_{N}$. This threshold ranges from a few hundred gigaelectronvolts, in hypercolor scenarios, to about $10^{10} \mathrm{GeV}$, in axion models, and all the way up to $10^{15} \mathrm{GeV}$, in typical grand unified theories.

It is very important to constrain the scale $M_{N}$. Experiments place lower limits on $M_{N}$ that are typically of the order of a few hundred gigaelectronvolts. The precise bounds depend on the model under consideration and on the values of various free parameters. In this Letter we describe a new upper bound of the scale $M_{N}$. We shall show that heavy quarks provide a model-independent probe of the $\mathrm{SU}(3) \otimes \mathrm{SU}(2)$ $\otimes \mathbf{U}(1)$ desert.

Our idea is based on the following two observations:

(1) Heavy fermions have large Yukawa couplings at the weak scale $M_{W}$. The structure of the $S U(3) \otimes S U(2) \otimes U(1)$ renormalization-group equations implies that such couplings blow up at a relatively low energy $M_{B}$.

(2) At the scale $M_{B}$, where the Yukawa couplings diverge, new physics should be found. For example, large Yukawa couplings induce strong couplings in the Higgs sector. Such a strongly coupled Higgs sector should give rise to a new spectrum of bound states and to new effective interactions. Of course, it is always possible that the gauge group might change at an ener- gy $M_{N}<M_{B}$. In this case, new gauge bosons of mass $M_{N}$ should then be found. No matter what happens, new physics must emerge before the Yukawa couplings diverge.

The idea that diverging couplings imply new physics is not new. In quantum electrodynamics, it is associated with the presence of "Landau ghosts." In QED, the electromagnetic coupling blows up at an astronomically high energy, about $M_{B} \geq e^{137} \mathrm{GeV}$. If QED were a fundamental theory, it would-at the very least-become strongly coupled at the scale $M_{B}$. New physics would arise, and quantum electrodynamics as we know it would break down. Of course, we can never see this new physics because QED is only an effective theory. At the scale $M_{N} \simeq 80 \mathrm{GeV}$, QED loses its separate identity and becomes absorbed into the standard electroweak theory. ${ }^{1}$

In what follows we apply these ideas to the standard $S U(3) \otimes S U(2) \otimes U(1)$ model. For ease of presentation, we first examine a three-family model with a heavy top quark (and a single Higgs doublet). We denote the top Yukawa coupling by $g_{t}$. Its evolution with energy is given by ${ }^{2}$

$$
d g_{t} / d \tau=\frac{1}{2} g_{t}\left(9 g_{t}^{2}-2 G\right),
$$

where $\tau=\ln \left(E / M_{W}\right) / 16 \pi^{2}, M_{W}=80 \mathrm{GeV}$, and $G$ $=8 g_{3}^{2}+2.25 g_{2}^{2}$. Here $g_{3}$ and $g_{2}$ are the strong and weak gauge couplings, and we neglect small hypercharge effects. As can be seen in Eq. (1), the evolution of $g_{t}$ has two types of contributions. The first comes from Higgs boson exchanges and tends to increase the Yukawa coupling at high energies. The second comes from the gauge bosons; it tends to decrease the Yukawa coupling. For large values of $g_{t}$, the first term dominates, and the Yukawa coupling grows exponentially with energy. 


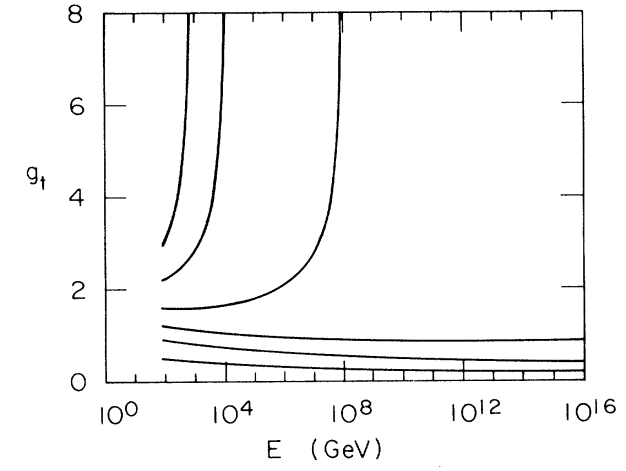

FIG. 1. The evolution of the top Yukawa coupling $g_{t}$ as a function of the energy $E$. We have evolved the color gauge coupling using $\Lambda_{\overline{\mathrm{MS}}}=250 \mathrm{MeV}$.

In Fig. 1 we show the evolution of $g_{t}$ with energy for a variety of initial conditions $g_{t}^{0}$ (at the weak scale $\left.M_{W}\right)$. For $g_{t}{ }^{0}>2 G / 9$, the coupling $g_{t}$ increases with energy and diverges at an energy $M_{B} \cdot{ }^{3}$ Because of Eq. (1), it diverges faster and faster for larger and larger initial conditions.

In Fig. 2 we plot the scale $M_{B}$ as a function of the top mass $m_{t}$ (evaluated at the weak scale $M_{W}$ ). With our conventions, $m_{t}=g_{t}{ }^{0}\langle\phi\rangle$, where $\langle\phi\rangle$ is normalized to $175 \mathrm{GeV}$. For large initial conditions, the scale $M_{B}$ is quite small. A top mass of $250 \mathrm{GeV}$ implies new physics below $M_{B}=10^{11} \mathrm{GeV}$. For $m_{t}=300 \mathrm{GeV}$, this tightens to just $10^{6} \mathrm{GeV}$. If $m_{t}$ is as large as 400 $\mathrm{GeV}$, new physics should appear below $10 \mathrm{TeV}$ ! The top mass provides a very sensitive probe of the $S U(3) \otimes S U(2) \otimes U(1)$ desert.

The value of $M_{B}$ depends sensitively on the value of $g_{3}$ at the weak scale $M_{W}$. The larger the value of $g_{3}$, the larger the scale $M_{B}$. Since we are bounding $M_{N}$ from above, we wish to choose the maximum value of $g_{3}$ consistent with experiment. Therefore, we take $g_{3}$

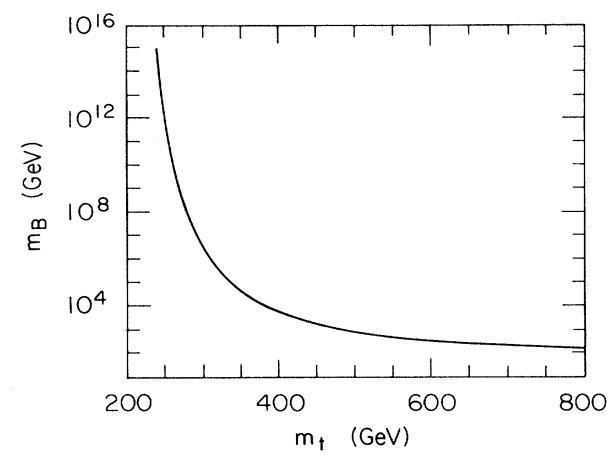

FIG. 2. The upper limit on the scale of new physics, as a function of the top mass $m_{t}$ in a three-family model. As in Fig. 1 , we take $\Lambda_{\overline{\mathrm{MS}}}=250 \mathrm{MeV}$. Smaller values of $\Lambda_{\overline{\mathrm{MS}}}$ lead to tighter bounds. to be given by $\Lambda_{\overline{\mathrm{MS}}}=250 \mathrm{MeV}$, in the four-flavor regime ( $\overline{\mathrm{MS}}$ denotes the modified minimal-subtraction scheme).

More general bounds on $M_{N}$ can be found by examination of the renormalization of an arbitrary number of heavy families. The appropriate generalization of the top Yukawa coupling is the so-called quark "radius" $T_{Q}^{2}=\Sigma g_{q}^{2}$. Here the $g_{q}$ denote the (real) eigenvalues of the quark Yukawa matrices, and the sum runs over all up- and down-type quarks. The renormalization of $T_{Q}$ has been investigated elsewhere. ${ }^{4}$ For large Yukawa couplings, the quark radius renormalizes even more quickly than the individual couplings themselves.

As with the top Yukawa coupling, the evolution of $T_{Q}$ can be used to bound the scale of new physics. Instead of $m_{t}$, we now plot $\left(\Sigma m_{q}^{2}\right)^{1 / 2}$, where the index $q$ runs over all up- and down-type quarks. The results are shown in Fig. 3. When $\sum m_{q}^{2}>(355 \mathrm{GeV})^{2}$, $M_{N}<10^{15} \mathrm{GeV}$. The bound tightens very quickly. For $\Sigma m_{q}^{2}=(450 \mathrm{GeV})^{2}$, the scale $M_{N}$ lowers to just $10^{5} \mathrm{GeV}$. Because of the renormalization group, the heavy-quark spectrum provides a model-independent probe of the $\mathrm{SU}(3) \otimes \mathrm{SU}(2) \otimes \mathrm{U}(1)$ desert. If the sum $\Sigma m_{q}^{2}$ is large, the desert must bloom.

In Fig. 3, we have evolved $g_{2}$ and $g_{3}$ with the beta functions appropriate for $N_{F}=8,9,10$, and 11 families. The limits for $N_{F}<8$ are not shown because they are even tighter. At any given energy, the bound on $M_{N}$ is given by the curve with the greatest value of $N_{F}$. In Fig. 3, the graphs for $N_{F}>8$ are terminated when the color coupling blows up.

Certain extensions of the standard model have two Higgs doublets at low energies. These models usually have one Higgs doublet coupled to up-type quarks, and the other coupled to their down-type partners. This

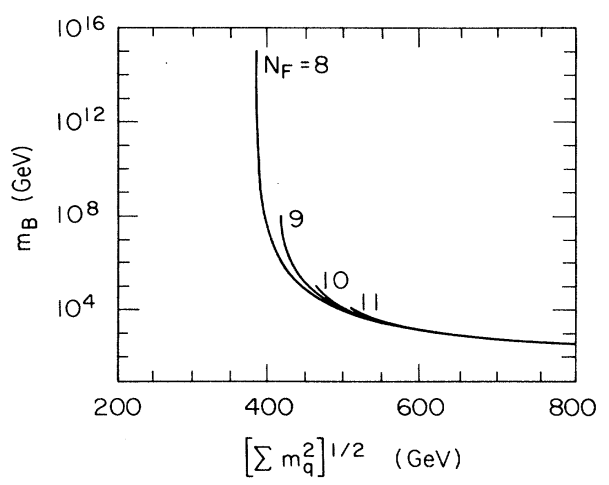

FIG. 3. The upper limit on the scale of new physics, as a function of the quark radius $\left(\Sigma m_{q}^{2}\right)^{1 / 2}$. The bound is given by the graph with the greatest number of families in a given energy region. We have included results for $N_{F}=8,9,10$, and 11. The graphs terminate when the color coupling diverges. As before, we take $\Lambda_{\overline{\mathrm{MS}}}=250 \mathrm{MeV}$. 


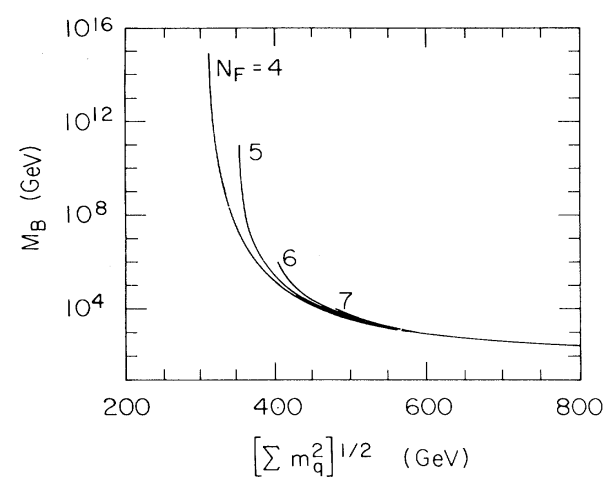

FIG. 4. The same as in Fig. 3, for the supersymmetric version of the standard model. We include the limits for $N_{F}=4,5,6$, and 7 .

type of coupling naturally suppresses flavor-changing neutral currents. Our analysis is easily extended to include these two-Higgs-doublet models. The evolution of $T_{Q}$ differs from the one-Higgs-doublet case, but it is not hard to see that the bounds on $M_{N}$ remain the same. ${ }^{5,6}$ Therefore, the limits in Fig. 3 are also valid in two-Higgs-doublet extensions of the standard $\mathrm{SU}(3) \otimes S U(2) \otimes U(1)$ model.

Finally, we consider the supersymmetric extension of the standard model. We shall see that if supersymmetry is found below the weak scale $M_{W}$, the bounds on $M_{N}$ can be tightened still further. This follows from the fact that the superpartners of the quarks, leptons, and gauge bosons all contribute to the $S U(3) \otimes S U(2) \otimes U(1)$ beta functions. ${ }^{7}$ The final results are shown in Fig. 4, with the assumption that the superpartners are all lighter than $80 \mathrm{GeV}$. As before, we take $\Lambda_{\overline{\mathrm{MS}}}=250 \mathrm{MeV}$. Because of the effects of the superpartners, the color beta function blows up very quickly, and so Fig. 4 includes only the four cases $N_{F}=4,5,6$, and 7 .
In this Letter we have demonstrated that heavy quarks provide a model-independent probe of the desert. They extend the range of teraelectronvolt accelerators to well beyond the center-of-mass energies. The discovery of new heavy quarks would indicate that new physics waits to be found in the SU(3) $\otimes \mathrm{SU}(2) \otimes \mathrm{U}(1)$ desert.

This work was supported by the U. S. Department of Energy through Contract No. DE-AC03-76SF00515, and by the National Science Foundation through Contract No. NSF-PHY-83-10654. One of us (S.D.) is a recipient of an Alfred P. Sloan Foundation fellowship.

(a) Permanent address: Departament de Física Teòrica,
Universitat Autonoma de Barcelona, Bellaterra, Spain.
1The hypercharge factor in the SU(3) $\otimes S U(2) \otimes U(1)$
model also suffers from Landau ghosts. In grand unified model also suffers from Landau ghosts. In grand unified $\otimes S U(2) \otimes U(1)$ in an asymptotically free semisimple gauge group $G$.

${ }^{2}$ T. Cheng, E. Eichten, and L. Li, Phys. Rev. D 9, 2255 (1974).

${ }^{3} \mathrm{We}$ run the gauge couplings with their two-loop renormalization-group equations. $M_{B}$ is defined to be the scale where $\alpha_{t}=g_{t}{ }^{2} / 4 \pi=10$.

${ }^{4}$ B. Pendleton and G. Ross, Phys. Lett. 98B, 291 (1981); C. Hill, Phys. Rev. D 24, 691 (1981); E. Paschos, Z. Phys. C 26, 235 (1984); J. Bagger, S. Dimopoulos, and E. Massó, Nucl. Phys. B253, 397 (1985).

5 J. Bagger, S. Dimopoulos, and E. Massó, Phys. Lett. 156B, 357 (1985).

${ }^{6}$ The second doublet gives a small contribution to the renormalization of $g_{2}$. We neglect this effect; it leaves our bounds essentially unchanged.

${ }^{7}$ The evolution of $T_{Q}$ in the supersymmetric case is discussed in J. Bagger, S. Dimopoulos, and E. Massó, Phys. Rev. Lett. 55, 920 (1985). 